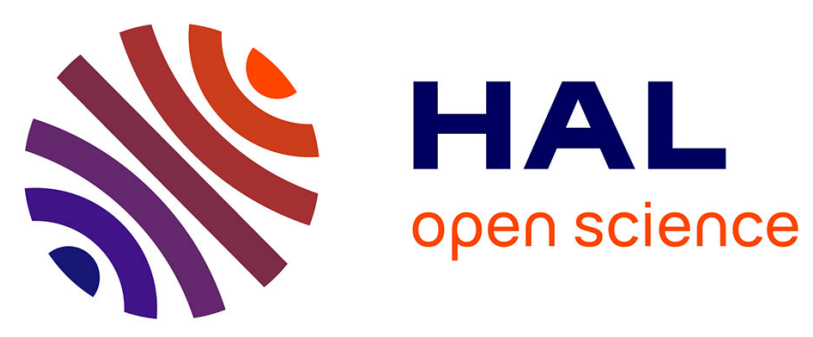

\title{
Cluster analysis of acoustic emission data to investigate the damage evolution in modified scarf joint under bi-axial loading
}

Jaime Destouesse, Malick Diakhate, Claudiu Badulescu, David Thevenet, Matthieu Stackler, William Albouy, Nicolas Carrere

\section{To cite this version:}

Jaime Destouesse, Malick Diakhate, Claudiu Badulescu, David Thevenet, Matthieu Stackler, et al.. Cluster analysis of acoustic emission data to investigate the damage evolution in modified scarf joint under bi-axial loading. Journal of Adhesion, 2020, 96 (11), pp.969-987. 10.1080/00218464.2018.1552854 . hal-02052697

\section{HAL Id: hal-02052697 \\ https://hal-ensta-bretagne.archives-ouvertes.fr/hal-02052697}

Submitted on 27 Sep 2021

HAL is a multi-disciplinary open access archive for the deposit and dissemination of scientific research documents, whether they are published or not. The documents may come from teaching and research institutions in France or abroad, or from public or private research centers.
L'archive ouverte pluridisciplinaire HAL, est destinée au dépôt et à la diffusion de documents scientifiques de niveau recherche, publiés ou non, émanant des établissements d'enseignement et de recherche français ou étrangers, des laboratoires publics ou privés. 


\title{
Cluster analysis of acoustic emission data to investigate the damage evolution in modified scarf joint under bi-axial loading
}

\author{
J. Destouesse ${ }^{\mathrm{a}, \mathrm{b}}$, M. Diakhate $\mathbb{C}^{\mathrm{c}}$, C. Badulescu ${ }^{\mathrm{b}}$, D. Thévenet ${ }^{\mathrm{b}}$, M. Stackler ${ }^{\mathrm{a}}$, \\ W. Albouy ${ }^{a}$, and N. Carrere ${ }^{a}$ \\ aSafran Composites, Itteville, France; ${ }^{b} E N S T A$ Bretagne, IRDL-UMR CNRS 6027, Brest, France; 'Univ. \\ Bretagne Occidentale, IRDL-UMR CNRS 6027, Morlaix, France
}

\begin{abstract}
Non-destructive monitoring of damage evolution within mate-rial or bonding assembly becomes an essential tool to better understand its mechanical behavior, and therefore to prevent failure risks of engineering structures that involve adhesive bonding matters. This paper presents the experimental results of monotonic tests that were conducted firstly to investigate the effects of bi-axial loadings (with different shear/peel ratios) on the mechanical damage evolution of metal/metal bonded joint, and secondly to both detect and identify the acoustic emission (AE) signatures of the different failure mechanisms involved in the bonded joint damage. Results from specimens with modified scarf joint show that the loading configuration (shear/peel ratio) strongly influences the normal stiffness of the adhesively-bonded joint. For each loading configuration, repe-titive tests were performed, and loading rate effects on the mechanical behavior of adhesively-bonded joint were ana-lyzed. In addition to these results, a k-means++ algorithm was used to achieve a cluster analysis of $\mathrm{AE}$ data, and to allow $\mathrm{AE}$ events that were generated by damage evolution of the bonded joint to be identified. A particular AE signature is highlighted since it allows monitoring damage evolution of the adhesively-bonded joint. Test results also show that the high-est value of acoustic energy is detected when the slope of the mechanical behavior curve (macroscopic scale) drastically changes. This finding is used to perform a real-time detection of the adhesive yield strength.
\end{abstract}

\section{Introduction}

Over the last decades adhesive bonding has found many engineering applications such as marine, automobile, aerospace and construction. ${ }^{[1-3]}$ The joining of two or more components allows for structures to perform their operational requirements, transferring forces from one surface to another. 
The choice of this assembly method is mainly due to the advantages that adhesive bonding provides over traditional mechanical fixing techniques (bolting, welding, and screwing) including weight reductions, lower manufacturing cost, the variety of materials that can be bonded, a uniform stress distribution of the load, better fatigue properties, etc.

To further improve the performance and safety of adhesively bonded joints, a deeper understanding of their behavior in relation to the loading conditions is crucial. From literature, two major failure types are usually observed at the surface of the bonded joint: adhesion failure, slick failure at the interface between the adhesive and the adherent surface, and cohesion failure, fracture within the adhesive material itself.

In a mechanical structure, the adhesive joint may be subjected to multiaxial loadings. In laboratory, investigating the adhesive joint mechanical properties under this stress state led some researchers to propose specific test devices such as the modified Arcan fixture. ${ }^{[4,5]}$ However, it's not easy to use such devices in an industrial environment, and the use of a large range of non-destructive techniques is limited. This study consists in the development of the modified scarf test which allows the implementation of different monitoring techniques. It has the advantage that it can be easily performed with a tensile test machine in an industrial environment, and it also allows applying multiaxial loadings without having high stress concentrations near the edges.

Although many monitoring procedures have been used in field or lab conditions in order to analyze appears failure within adhesive joints, such as radiography, infrared spectroscopy ${ }^{[6-9]}$ and ultrasonic method, which is widely used for monitoring the structural health of components, ${ }^{[1,10,11]}$ only few of them have proven their efficiency. In addition, the ultrasonic sensors must be coupled to the structure following a specific procedure, which can be time-consuming. Acoustic emission (AE) method can be used to detect transient elastic waves emitted by a growing crack within the material. ${ }^{[12-}$ ${ }^{16]} \mathrm{AE}$ can be used in the identification of failure at extremely early stages, thus preventing severe structural damage. Several authors have attempted to identify $\mathrm{AE}$ signal signatures from various fracture mechanisms of materials ${ }^{[8,17-20]}$ or adhesive composite joints, ${ }^{[21,22]}$ using computational pattern recognition approaches, the studies showed that the $\mathrm{AE}$ signal were directly related to failure mechanism.

The aim of this study is, on the one hand, to investigate the mechanical behavior of a thin thickness adhesively-bonded joint under multiaxial loadings using a new specimen, showing that this test might also be used for the characterization of the adhesive behavior considering time effects (fatigue or creep), and on the other hand, to build a monitoring protocol (based on the $\mathrm{AE}$ method) that allows real-time detection and identification of the different failure mechanisms associated with the mechanical behavior of bonded 
joints. An unsupervised classification method (based on k-means++ algorithm) is proposed to identify AE signatures of those failure mechanisms.

\section{Experimental procedure}

This section describes the mechanical tests conducted to investigate the mechanical behavior of an adhesively-bonded joint under bi-axial loadings, but also the ability of using the AE technique to detect, locate, and monitor the different failure mechanisms within the adhesive material. The experimental conditions (specimen preparation, instrumentation techniques, and mechanical loading conditions) are described.

\subsection{Modified scarf specimen}

Figure 1 presents the geometry of the modified Scarf specimens inspired from Scarf joints. ${ }^{[23]}$ Arms and beaks have been added in order to have homogeneous stress distributions along the surface and to reduce the stress concentrations near the free edges as proposed by Cognard et al. for the Arcan fixture. ${ }^{[5]}$ The overlap length $\left(L_{n}\right)$ is a function of the angle $\delta$ of the modified Scarf joint, and is equal to $L_{n}=\frac{L}{\operatorname{Cos}(\delta)}+2 \times L_{\text {arm }}$. The angle $\delta$ at the middle branch of modified Scarf specimens can be changed in order to apply different bi-axial loadings within the adhesive joint. In this work, three loading configurations (different angles $\delta$, Figure 1) were tested: $\delta=0^{\circ}, 22.5^{\circ}$, and $45^{\circ}$. Table 1 presents the stress (tensile and shear) levels that are associated with each loading configuration.

\subsection{Materials and specimen preparation}

The modified scarf joint specimens are manufactured using Aluminum alloy AW7075 T6 and high pressure water jet cutting. Surfaces to be bonded were ground with a $220 \mathrm{SiC}$ abrasive paper and cleaned with acetone solution to remove the remaining residues; a structural epoxy adhesive film was applied over the surfaces. In order to ensure the better positioning of substrates (two half-scarf specimens), a bonding device has been designed and manufactured (Figure 2).

The planarity of the specimen ( $\mathrm{z}$ direction) is ensured thanks to the bonding table. The top cylinders and the top grid ensure the right positioning of the top substrates. The bottom substrate is positioned thanks to the bottom cylinder. The adhesive joint thickness is ensured using a calibrated metal wire of $200 \mu \mathrm{m}$, which is located at the right and left extremities of the arms (where the stresses tend to zero). Finally the modified scarf joint specimen was cured following the manufacturer recommended cycle of the adhesive material. 


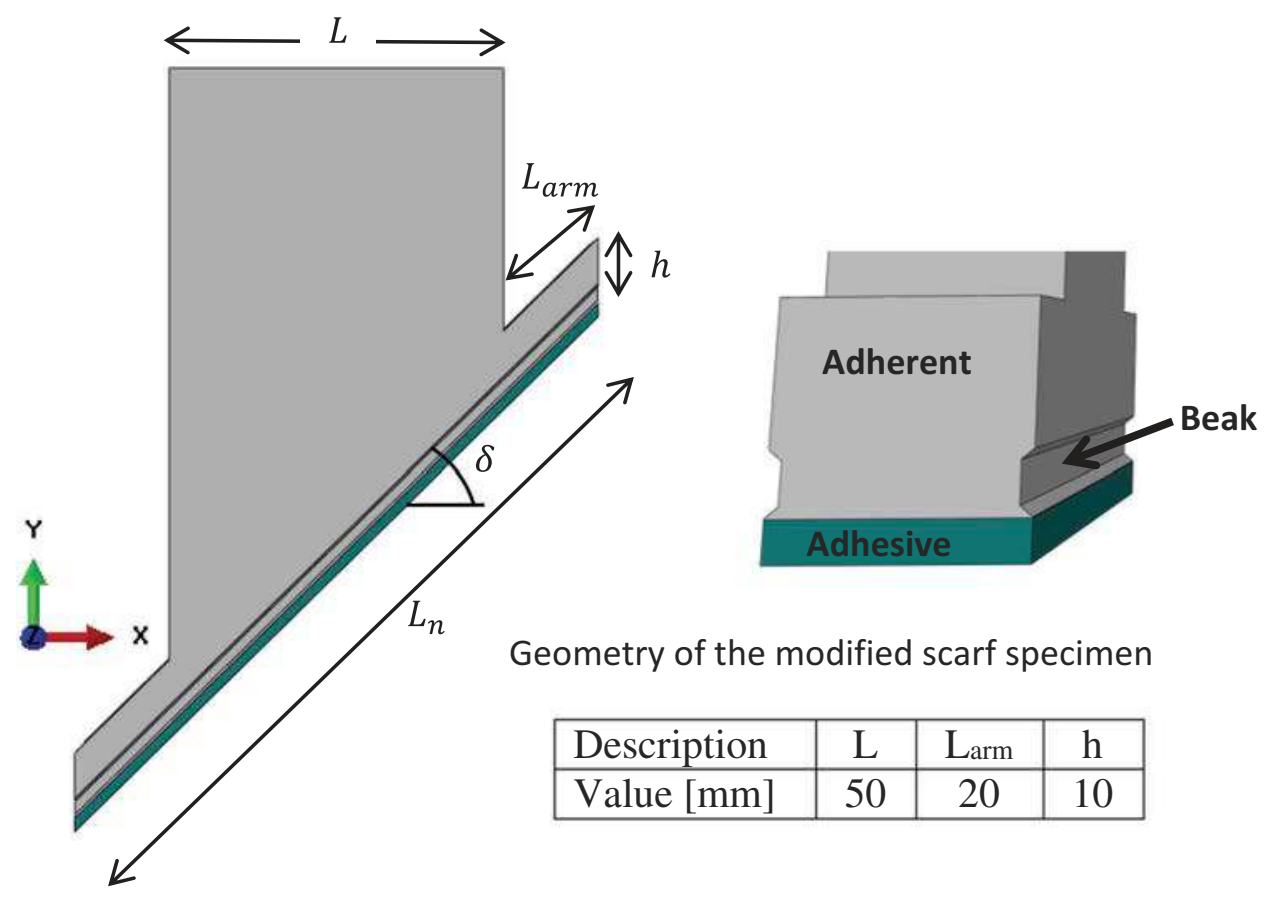

Figure 1. Shape of the improved scarf joint proposed in ${ }^{[20]}$.

\subsection{Instrumentation techniques: DIC and $A E$}

In this work, stereo Digital Image Correlation (DIC) method was used for measuring both the normal and the tangential relative displacements as a function of the applied load, along the adhesively-bonded joint. The DIC equipment (GOM Optical Measuring Techniques ARAMIS 3D (Widen, Switzerland)) consists of two 5M digital CCD cameras (Figure 3), a lighting system, and a data acquisition system. Calibration of CCD cameras was performed using a $25 \mathrm{~mm} \times 20 \mathrm{~mm}$ standard to obtain a resolution deviation of less than $0.01 \mu \mathrm{m}$. In this study, the images were recorded at a sampling frequency of $2 \mathrm{~Hz}$. The computation of the displacement field in both directions was accomplished as the difference between two zones close to the adhesive joint and symmetrical about the midplane of the bonded layer (Figure 4a). Each displacement is obtained in a local coordinate system that depends on the angle $\delta$ of the modified Scarf specimen Figure $4 \mathrm{~b}$.

Under mechanical loading, cracking within the adhesive bond generates transient elastic waves, referred in this paper as AE waveforms. Thus, in addition to the DIC equipment, a four-channel AE system designed by MISTRAS Group (New Jersey, United States) was used to record the AE waveforms within the adhesive joint during the tests. Since the crack path within the adhesively bonded joint is known in advance, two AE channels 
Table 1. Summary of the stress distribution and levels with respect to the loading angle.

\begin{tabular}{lll}
\hline Angle $\delta\left(^{\circ}\right)$ & Tension stress level & Shear stress level \\
\hline 0 & ++++ (high) & Not applicable \\
22.5 & +++ (medium) & + (low) \\
45 & ++ (medium) & ++ (medium) \\
\hline
\end{tabular}

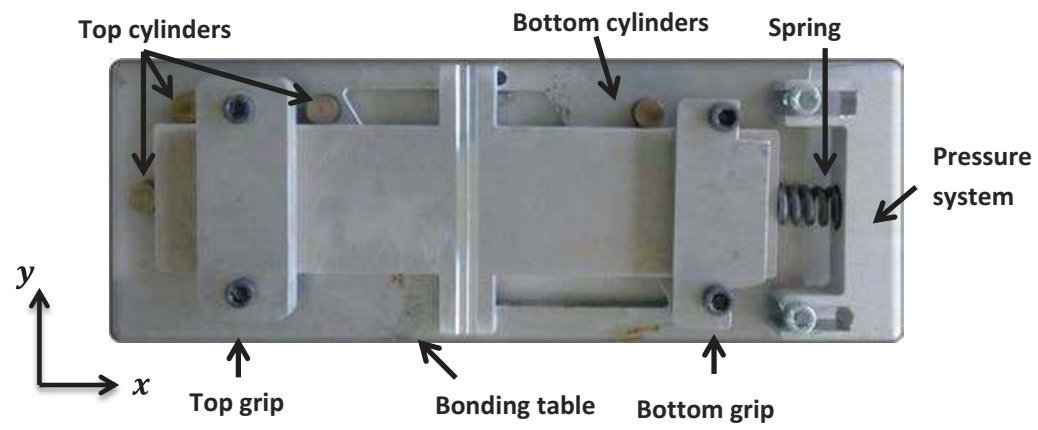

Figure 2. Device for bonding the two half-scarf specimens.

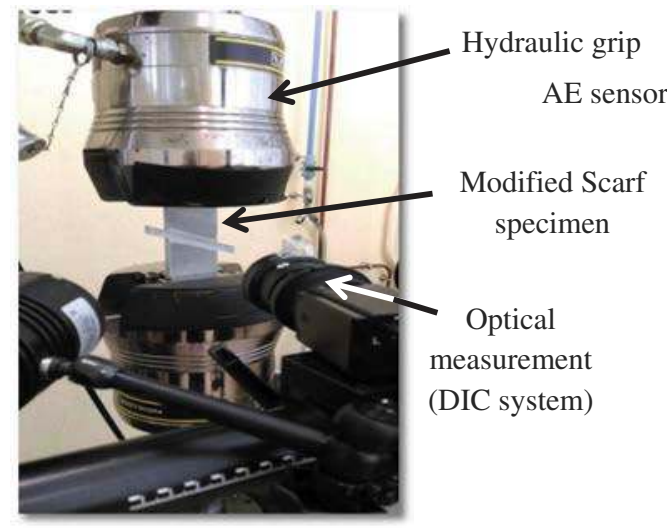

(a)

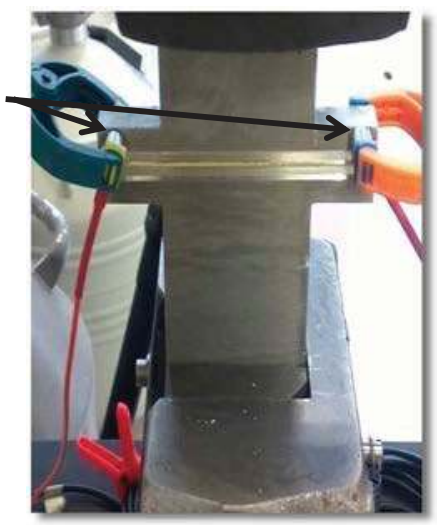

(b)

Figure 3. Instrumentation techniques: (a) DIC and (b) AE systems.

were used in order to perform a linear localization of the acoustic sources (or $\mathrm{AE}$ events). These two AE sensors (Figure 3), with optimum frequency range from $125-750 \mathrm{kHz}$ and a resonant frequency of $300 \mathrm{kHz}$, were connected to two preamplifiers, which were connected to the acquisition system. The AE sensors were coupled to the specimen upon the static adherent with silicon grease and held fixed in position with clamps. This procedure ensures a good acoustic coupling and also allows performing a linear localization (as shown in Figures 10 and 12) of the acoustic sources within the overlap length $\left(L_{n}\right)$.

As a result of using an Instron servo-hydraulic testing machine, hydraulic grips, and samples made of aluminum (Figure 3), acoustic signal acquisition 


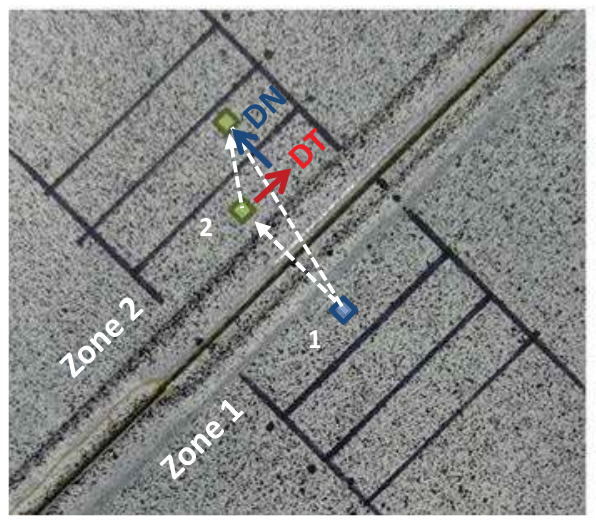

(a)

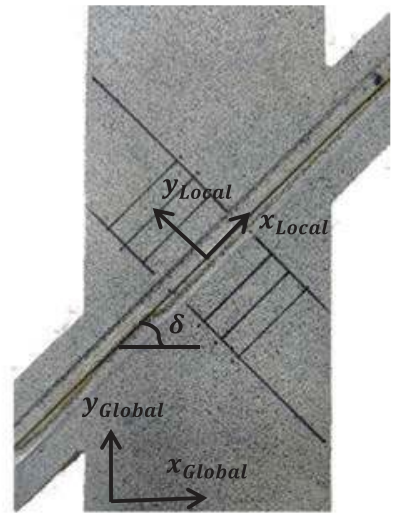

(b)

Figure 4. (a) Representative DIC image - (b) Scarf local coordinate system.

threshold was set at $55 \mathrm{~dB}$ in order to filter out vibrations induced by the testing machine. The sampled rate of $\mathrm{AE}$ waveforms was set at $40 \mathrm{MHz}$. Before mechanical tests, pencil lead breaking test was used to evaluate the acoustic wave propagation velocity along the bonding joint. ${ }^{[24,25]}$ The wave propagation velocity of $4000 \mathrm{~m} / \mathrm{s}$ was used in the linear localization of the AE sources within the adhesive joint.

Both systems were fully synchronized with the tensile machine so that, for each recorded image and for each acoustic emission event, the corresponding value of the applied force was also registered.

\subsection{Experimental protocol}

In order to investigate the adhesive mechanical behavior under different loading configurations $\left(0,22.5\right.$, and $\left.45^{\circ}\right)$, and at different loading rates $(0.02,0.2$, and $2 \mathrm{kN} / \mathrm{s})$, three specimens of each modified scarf joint were tested under force control until failure.

\section{Mechanical behavior of modified scarf joints}

In this section, experimental results on the effects of loading configuration and loading rates are presented and discussed. For confidentiality reasons, experimental results (relative displacements and force values) are normalized.

\subsection{Effects of loading configuration}

Experimental results showed that the highest load machine value is obtained with the $45^{\circ}$ modified scarf joint, whereas the highest normal relative displacement is obtained with the $0^{\circ}$ modified scarf joint. 
Figure 5 presents the normalized load as a function of the normalized normal relative displacement. The normal stiffness value of the adhesivelybonded joint can be computed from this curve. For each loading configuration, the applied load is normalized with respect to the highest load value ( $45^{\circ}$ modified scarf); the relative normal displacement value is normalized with respect to the highest one ( $0^{\circ}$ modified scarf).

It can be seen from Figure 5 that the loading configuration strongly influences the normal stiffness value of the bonded joint. This is probably due to the stress ratio (shear/peel) associated with each loading configuration (Table 1). In addition, differences in normal stiffness values may be due to the fact that the adhesive bonded surface (Figure 1) varies with the loading configuration. Specimen's geometries as well as experimental results have led to the following correlations (1):

$$
\frac{\mathrm{S}_{22.5^{\circ}}}{\mathrm{S}_{0^{\circ}}} \approx \frac{\mathrm{F}_{\mathrm{e} 22.5^{\circ}}}{\mathrm{F}_{\mathrm{e} 0^{\circ}}} \approx \sqrt{1.09} \text { and } \frac{\mathrm{S}_{45^{\circ}}}{\mathrm{S}_{0^{\circ}}} \approx \frac{\mathrm{F}_{\mathrm{e} 45^{\circ}}}{\mathrm{F}_{\mathrm{e} 0^{\circ}}} \sqrt{2}
$$

Where $S_{\delta^{\circ}}$ and $F_{e} \delta^{\circ}$ represent the adhesively-bonded surface and the yield strength values associated with the loading configuration $\delta$.

Repetitive tests were performed at $0.2 \mathrm{kN} / \mathrm{s}$ for each loading configuration. For each specimen, the load machine as well as the relative displacement is normalized with respect to their corresponding value at failure. Figure 6 presents the mechanical behavior of the adhesively-bonded joint under each loading configuration. Results show a good reproducibility of the test and also a low

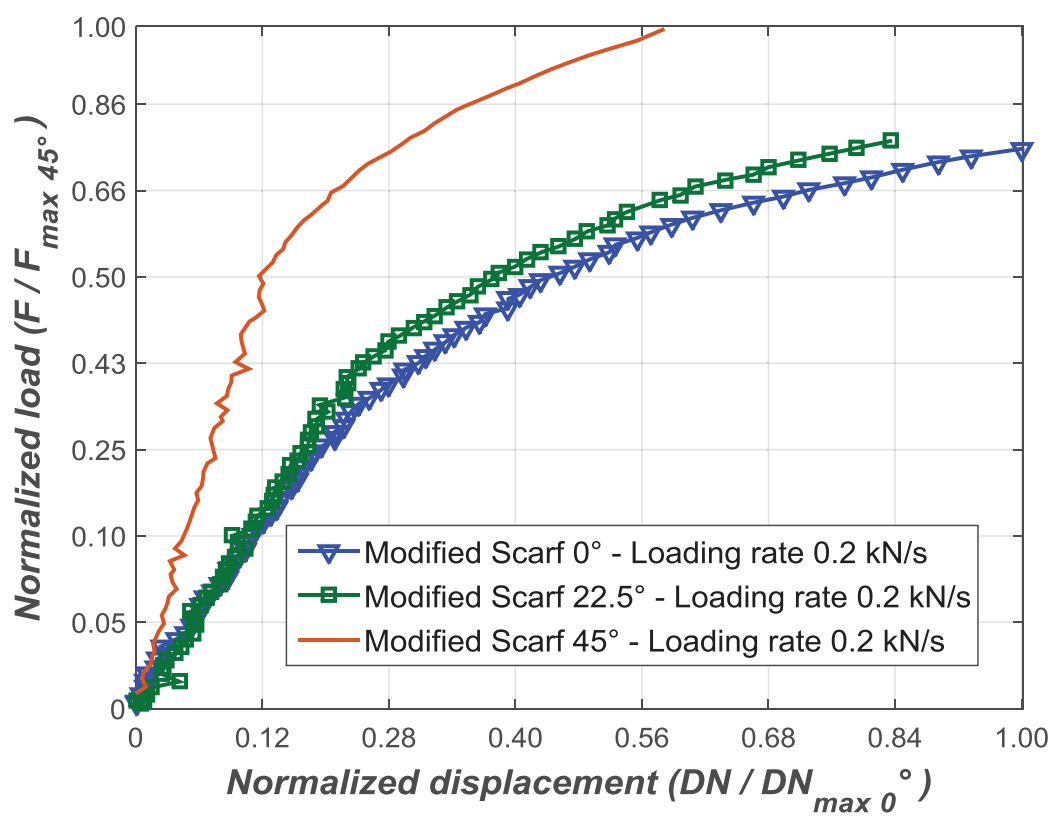

Figure 5. Effect of loading configuration on mechanical behavior of the modified scarf joint. 

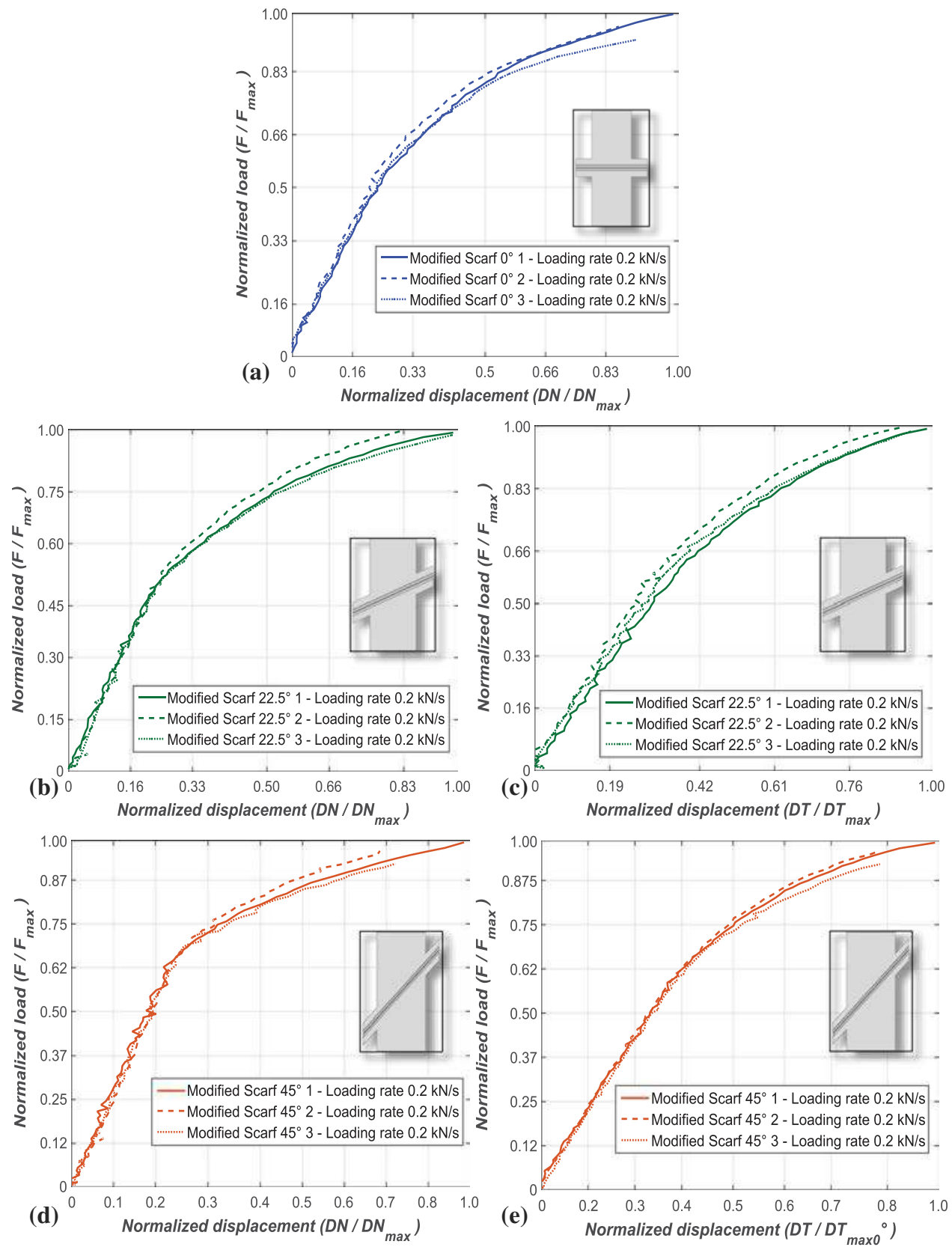

Figure 6. Repetitive tests at a loading rate of $0.2 \mathrm{kN} / \mathrm{s}$ : (a) Modified scarf $0^{\circ}-(\mathrm{b}$ and c) Modified scarf $22.5^{\circ}-\left(\mathrm{d}\right.$ and e) Modified scarf $45^{\circ}$.

scatter $(<2 \%)$ at each configuration of the modified scarf joint. The overall mechanical behavior shows two distinctive parts: the first one is characterized by a linear dependency between relative displacements and force machine, and the second one by a non-linear regime until the failure of the bonded joint. 


\subsection{Effects of loading rate}

As previously stated, the effect of loading rate on the adhesive mechanical behavior is investigated (Figure 7). For each specimen, load machine and relative displacements are normalized with respect to their corresponding values at failure. Experimental results show that the elastic behavior is not affected by the loading rate, whereas the non-linear regime as well as the load
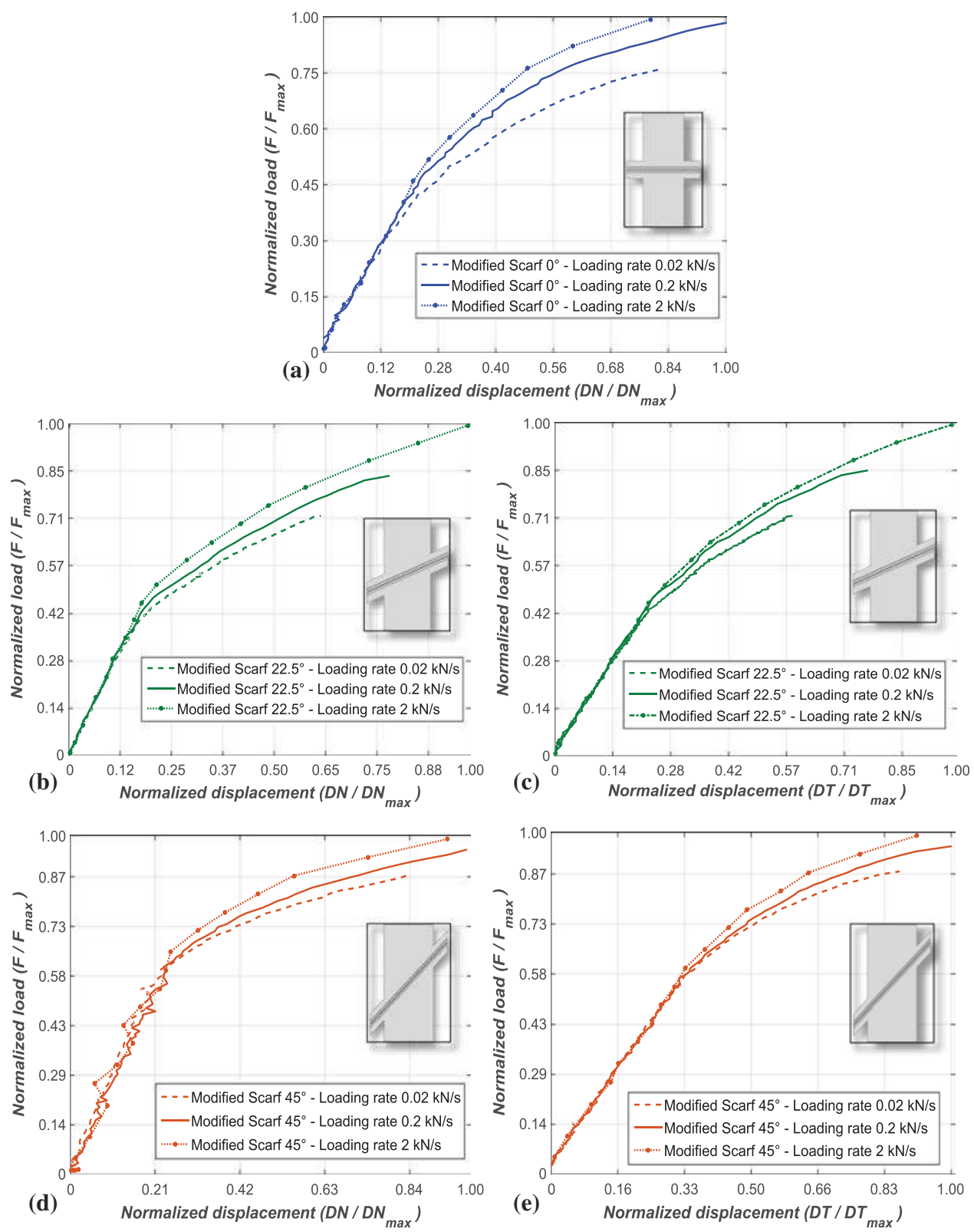

Figure 7. Effects of loading rate on mechanical behavior: (a) Modified scarf $0^{\circ}-(b$ and $c)$ Modified scarf $22.5^{\circ}-\left(\mathrm{d}\right.$ and e) Modified scarf $45^{\circ}$. 
machine at failure is strongly affected by the loading rate. In addition, it can be seen that the yield strength of the adhesively-bonded joint is not affected by the loading rate. Further on, we will discuss how the yield strength generates a particular acoustic signature (cf. paragraph 5.2).

\subsection{Fracture surfaces}

Representative fracture surfaces of two loading configurations $\left(0^{\circ}\right.$ and $45^{\circ}$ modified scarf joint) are presented in Figure 8. Optical observations using Keyence microscope pointed out cohesive failures for each $0^{\circ}$ modified scarf joint (Figure $8 \mathrm{a}$ ), whereas $22.5^{\circ}$ and $45^{\circ}$ modified scarf exhibit a mixed mode (cohesive and adhesive failures at the bonded surface, Figure $8 b-c$ ), and sharp ridges are locally observed.

\section{Damage mechanisms detection based on AE signatures}

As stated previously, cracking within the adhesive bond generates acoustic activity. This latter is analyzed with the aim of better understanding the failure mechanisms involved in the bonding joint damage. In a first part, the unsupervised classification method, which is used to both evaluate the number of failure mechanisms and identify their acoustic signatures, is detailed. In a second part, effects of loading configuration as well as mechanical loading rates on acoustic signatures of those failure mechanisms are investigated.

\subsection{Unsupervised classification method}

This method shows how the use of statistical tools (cluster algorithms) can help in gathering AE events into clusters (Figure 9). Among cluster algorithms, the most commonly used are k-means, self-organized map combination and fuzzy-C means algorithms. The K-means method is the most effective method for AE signal clustering, considering that the cluster

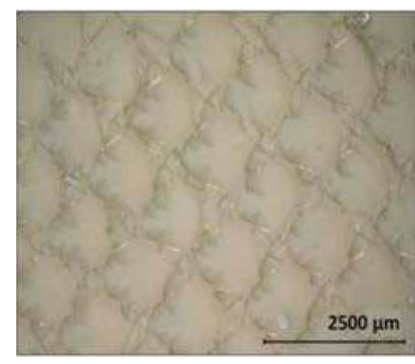

(a): Scarf $0^{\circ}$

(cohesive fracture)

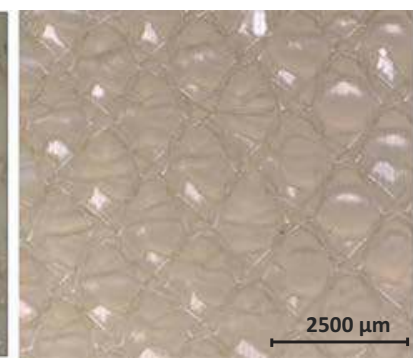

(b) : Scarf $22.5^{\circ}$

(adhesive /cohesive fractures) (adhesive / cohesive fractures)

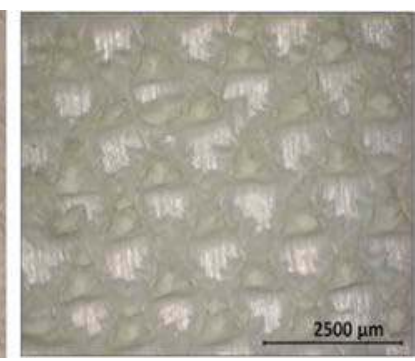

(c) : Scarf $45^{\circ}$

Figure 8. Effects of bonding configuration on the fracture surfaces. 

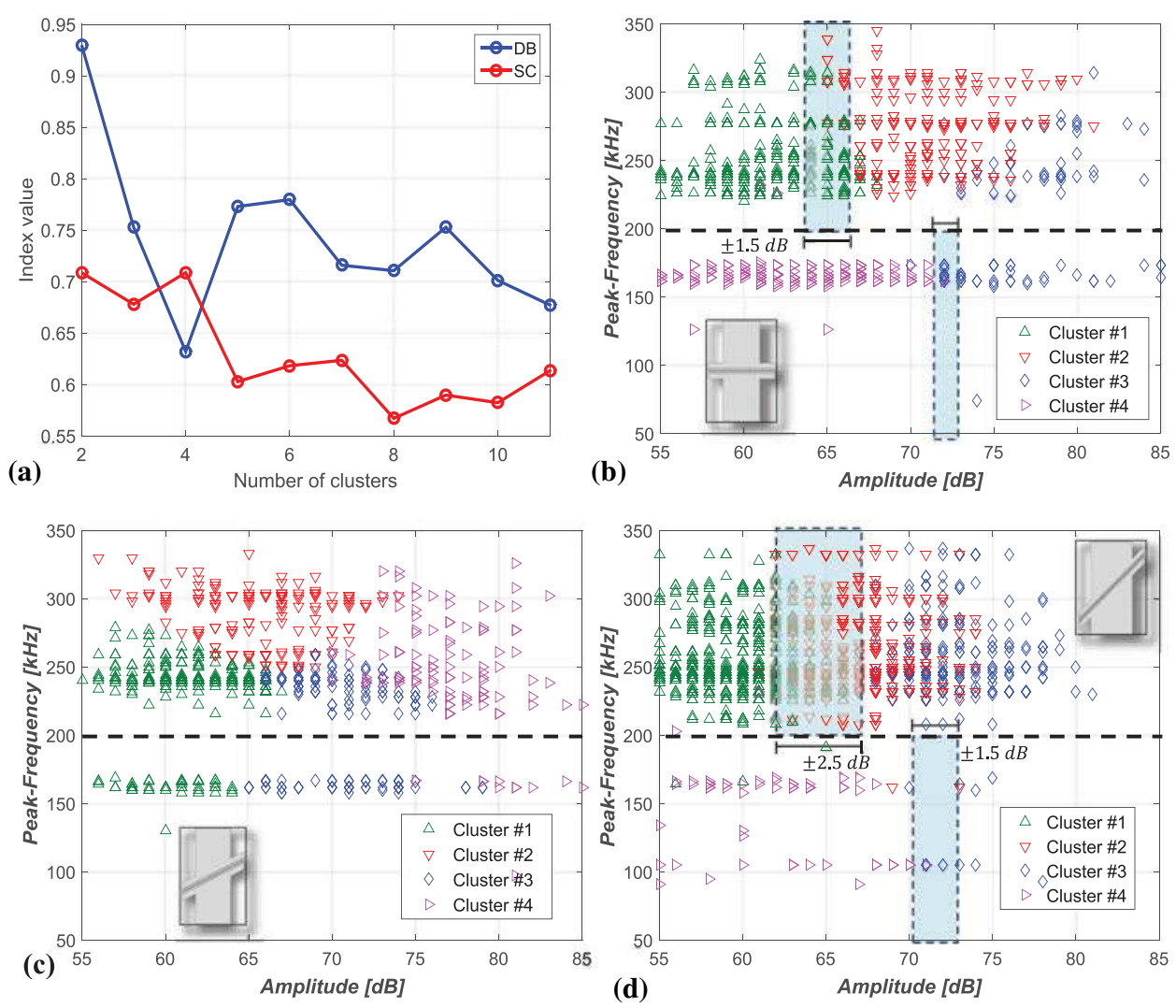

Figure 9. Clustering of $A E$ events using two $A E$ features: (a) optimal number, effect of loading configuration (b) $\delta=0^{\circ}$, (c) $\delta=22.5^{\circ}$, (d) $\delta=45^{\circ}$.

number is defined in advance from clustering evaluation indices (as discussed in section 4.2). ${ }^{[26]}$ This fact leads to more accurate minimization of the average squared distances between the points and the cluster center. In order to gather similar AE events into clusters, the k-means++ algorithm is combined with the statistical tool called Principal Component Analysis (PCA). ${ }^{[17,18]}$

\subsection{Cluster analysis results}

The first analysis to consider before gathering $\mathrm{AE}$ data into clusters is to eliminate the irrelevant and redundant signals from the AE features (amplitude, duration, energy, number of counts, rise time, and rise-amplitude, i.e. rise time divided by amplitude, the peak-frequency, the centroid-frequency, i.e. the center of gravity frequency, and the weighted-frequency). The selection of the most representative $\mathrm{AE}$ features for data clustering was achieved using the Laplacian score and the correlation coefficients. A detailed description of the Laplacian score is available in. ${ }^{[16]}$ 
In this study, the selection of the optimal number of clusters is based on the values of two clustering evaluation indices, the Davies-Bouldin index (DB) and the Silhouette Coefficient (SC). Additional details on both the DB and SC criteria are available in. ${ }^{[17,18]}$ The evaluation of these indices show that the optimal number of clusters is four ${ }^{\text {[27] }}$ (Figure 9a), which means that four cracking mechanisms with different acoustic signatures can be identified from the acoustic activity within the adhesive bonded joint.

For each loading configuration Scarf $0^{\circ}$ (Figure 9b), Scarf $22.5^{\circ}$ (Figure 9c), and Scarf $45^{\circ}$ (Figure 9d) a projection of the four clusters of AE events onto a two-dimensional plot is proposed. Results show that the loading configurations Scarf $0^{\circ}$ and Scarf $45^{\circ}$ allow identifying the clusters boundaries. Cluster analysis of $\mathrm{AE}$ events from Scarf $22.5^{\circ}$ do not allow obtaining well separated clusters.

Figure 9b-d show that amplitude and peak frequency show a good separation of the clusters points in the space. However, it should be kept in mind that peak frequency values of $\mathrm{AE}$ events are influenced by the resonant frequency of $\mathrm{AE}$ sensors. On this basis, Figure $9 \mathrm{~b}-\mathrm{d}$ led to classify the AE events into three main clusters. Separated by two borders at $65 \pm 1.5 \mathrm{~dB}$ and $73 \pm 0.5 \mathrm{~dB}$ for $0^{\circ}$ modified Scarf (for $45^{\circ}$ modified Scarf the borders are slightly larger): AE events of Cluster\#1 exhibit amplitude values lower than $65 \pm 1.5 \mathrm{~dB}$; AE events of Cluster\#2/Cluster \#3 (which are merged) exhibit amplitude values greater than $65 \pm 1.5 \mathrm{~dB}$; AE events of Cluster\#4 exhibit amplitude values lower than $73 \pm 0.5 \mathrm{~dB}$ (peak frequency within the range from 50 to $200 \mathrm{kHz}$ ).

Figure 10 shows the linear localization (between the two AE sensors, Figure 3) of $\mathrm{AE}$ events of the four clusters during the mechanical test for each loading configuration. It can be seen from these figures that the very first $\mathrm{AE}$ events belong to Cluster\#1 and Cluster\#2, and appear when the resulting force reaches half of its maximum value. AE events of Cluster\#3 start appearing when the resulting force reaches $0.6 \times \mathrm{F}_{\mathrm{Max}}$. Beyond this force value $\left(60 \%\right.$ of $\left.\mathrm{F}_{\max }\right), \mathrm{AE}$ events of all the clusters appear continuously along the adhesive bonded joint. It can also be seen from these figures that localization of Cluster\#3 (for Scarf $0^{\circ}$ and $45^{\circ}$ ) or Cluster\#4 (for Scarf $22.5^{\circ}$ ) $\mathrm{AE}$ events along the bonding joint and during the mechanical test follows a specific shape that is discussed in section 5.1.

\subsection{Effects of loading rates on AE activity}

Since mechanical tests with $0^{\circ}$ modified scarf joint generate acoustic activity with well separated clusters (Figure $9 \mathrm{~b}$ ), force-controlled tensile tests were performed on $0^{\circ}$ modified scarf specimens, and at different loading rates (Figure 11). This aims to investigate effects of these testing conditions on cluster analysis results of $\mathrm{AE}$ events. 

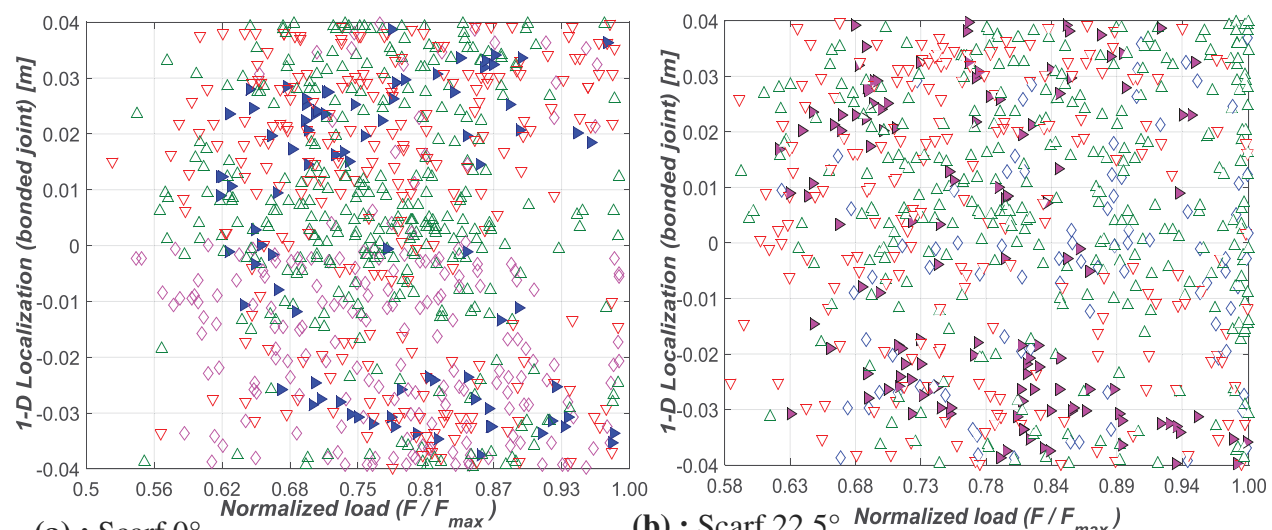

(a) : Scarf $0^{\circ}$

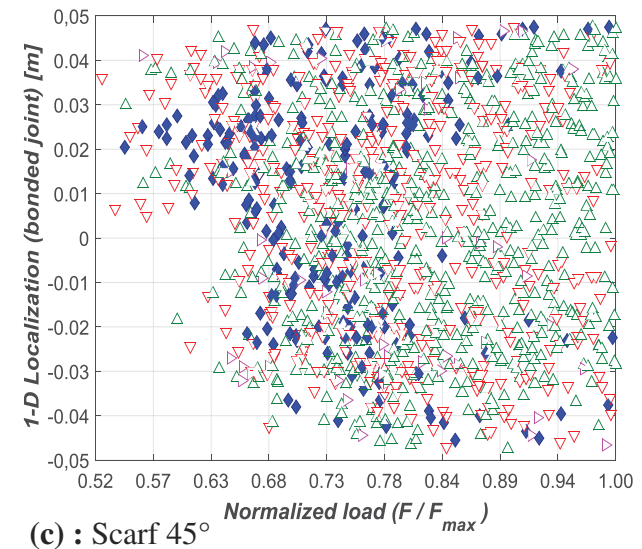

$\triangle$ Cluster \#1

$\nabla$ Cluster \#2

$\diamond$ Cluster \#3

$\triangleright$ Cluster \#4

(c) : Scarf $45^{\circ}$

Figure 10. Linear localization of AE events within the bonded joint: Effect of loading configuration.

When focusing on $\mathrm{AE}$ events with peak frequency within the range from 200 to $350 \mathrm{kHz}$, cluster analysis results show that under a low loading rate $(0.02 \mathrm{kN} / \mathrm{s})$ two main cracking mechanisms with different acoustic signatures can be identified (Figure 11a), and are differentiated by the amplitude level of $\mathrm{AE}$ events (lower or greater than $70 \mathrm{~dB}$ ).

\section{Correlation between AE activity and mechanical behavior}

In this section, the main goal is to perform an in-depth analysis of AE data in order to identify any acoustic signature allowing a detection of the mechanical behavior change during the modified scarf test. This change in the mechanical behavior (macroscopic) may be due to microstructural changes occurring within the adhesively-bonded joint. 

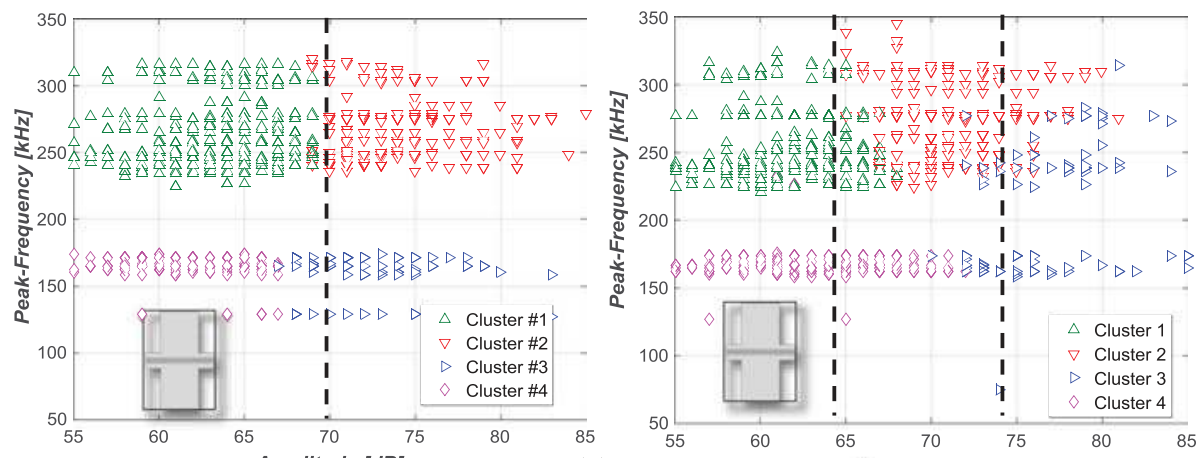

(a) : Test at $0.02 \mathrm{kN} / \mathrm{s} \quad$ Amplitude [dB]

(b) : Test at $0.2 \mathrm{kN} / \mathrm{s}$ Amplitude [dB]

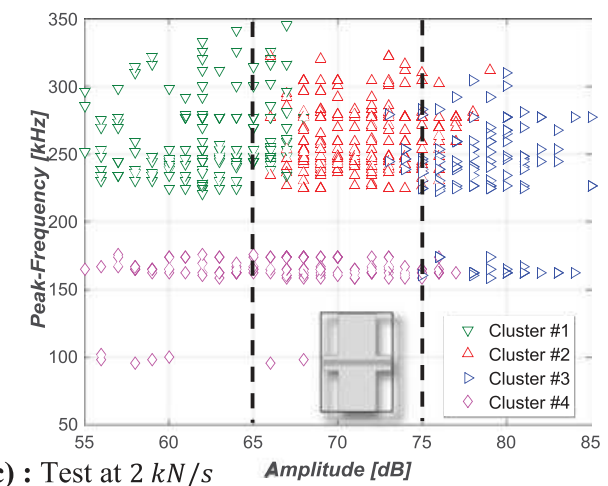

Figure 11. Clustering of AE events using two AE features: Effect of loading rate.

\subsection{Damage propagation monitoring}

As stated previously, the appearance of cluster\#3 AE events during mechanical test and along the adhesive bonding follows a specific shape (Figure 10). In particular, under loading configuration scarf $0^{\circ}$, a bell-shape distribution of cluster\#3 AE events is clearly noticeable (Figure 10a). This finding is used to evaluate the damage progress within the adhesive (Figure 12). As shown in Figure 12 damage levels of the bonded surface can be evaluated at different force values.

\subsection{Yield strength/damage detection}

This section will focus on the analysis of both the macroscopic mechanical behavior of the modified scarf joints and the acoustic emission activity within the adhesively-bonded joint. Acoustic energy is considered to be the AE feature that is highly correlated with mechanical strain energy. ${ }^{[17]}$

$\mathrm{AE}$ data that was recorded during mechanical tests on each loading configuration and at $0.2 \mathrm{kN} / \mathrm{s}$ is analyzed in terms of acoustic energy evolution (cumulative energy divided by cumulative number of events). As long as the yield strength of the material or the structure is not reached, the material 


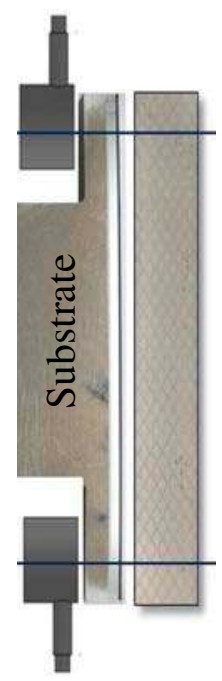

AE sensors

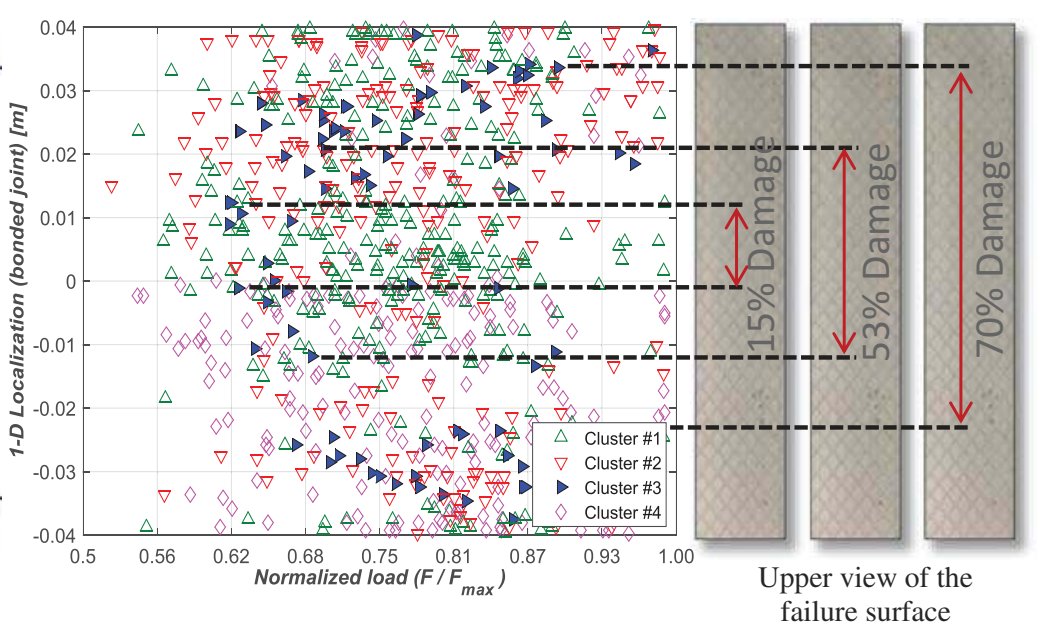

Figure 12. Damage surface evaluation by monitoring specific AE events.

continues to store mechanical energy. Once it is reached, the released strain energy is accompanied by transient elastic waves that are highly energetic, which can be related to permanent strain or micro-damage within the adhesive joint. During the test, AE software records the wave signals, and instantaneously computes and plots the corresponding acoustic energy values. This allows a real-time monitoring of acoustic energy evolution. Based on this evolution one can see from Figure 13 that the highest value of acoustic energy is detected when the slope of the mechanical behavior curve (macroscopic scale) drastically changes. This radical change in slope is generally assumed to correspond to the yield strength of the material.

In addition, it can be seen from Figure 13 that the very first $\mathrm{AE}$ events appear just before the adhesive bonded joint reaches its yield strength. These first $\mathrm{AE}$ events may be considered as warning signals. In order to identify the link between AE signals and permanent strain or damage, cyclic tests were performed using $0^{\circ}$ modified scarf specimens. The test was conducted by applying ten cycles with a step of $0.09 F_{\text {Max }}$. Figure 14 a shows the overlap between the linear localization of $\mathrm{AE}$ events and the applied load. It can be seen from these figures that the first big package of AE events appears after the 6 first cycles $\left(0.57 F_{M a x}\right)$. Also from Figure $14 \mathrm{~b}$ it can be seen that the unloading of the 6 first cycles has no permanent deformation, which leads to the conclusion that these events are related to micro-damage. Further analysis using tomography scans will allow identifying the defects that generate these particular acoustic signatures. 

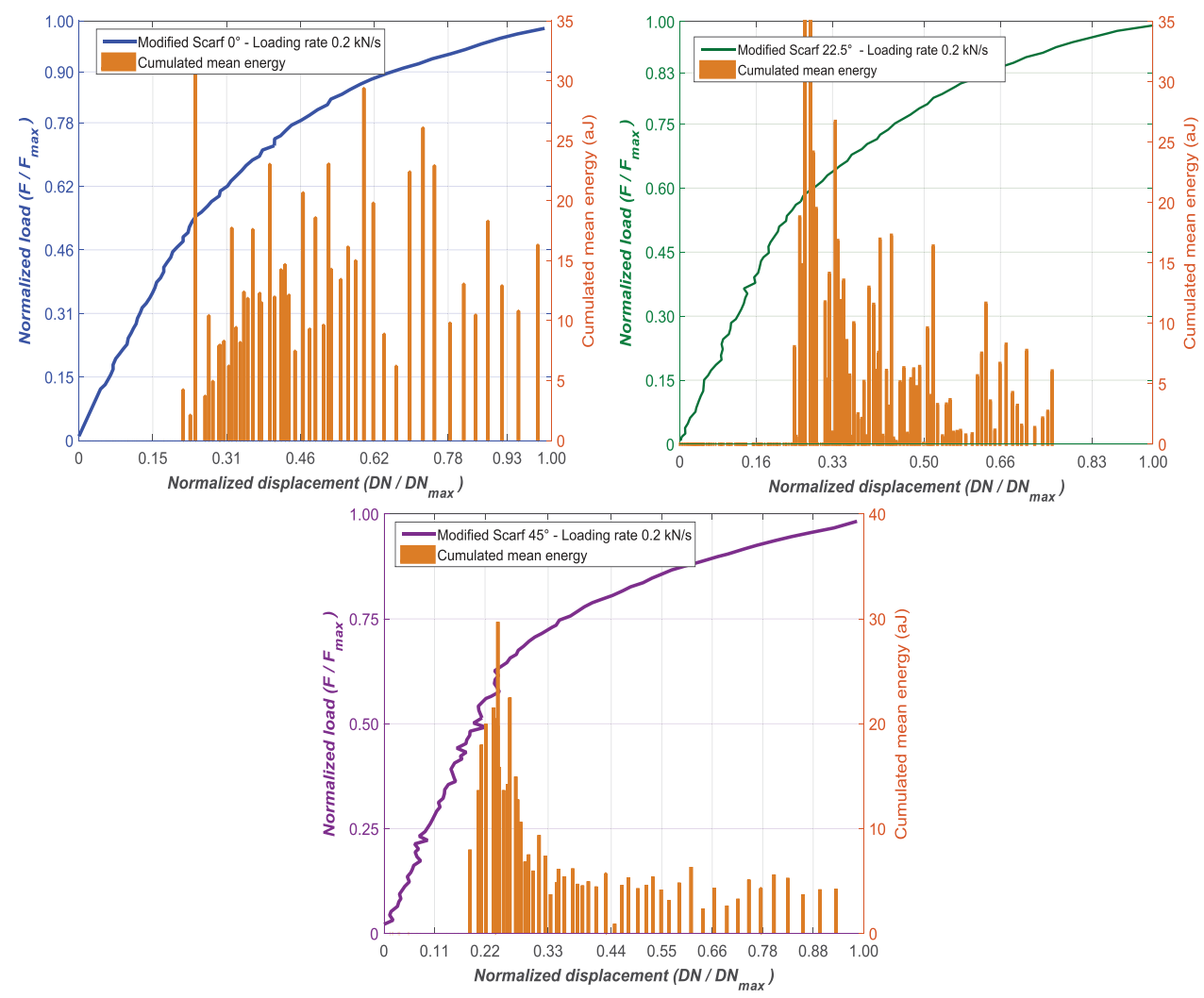

Figure 13. Real-time yield strength detection by means of acoustic emission energy.

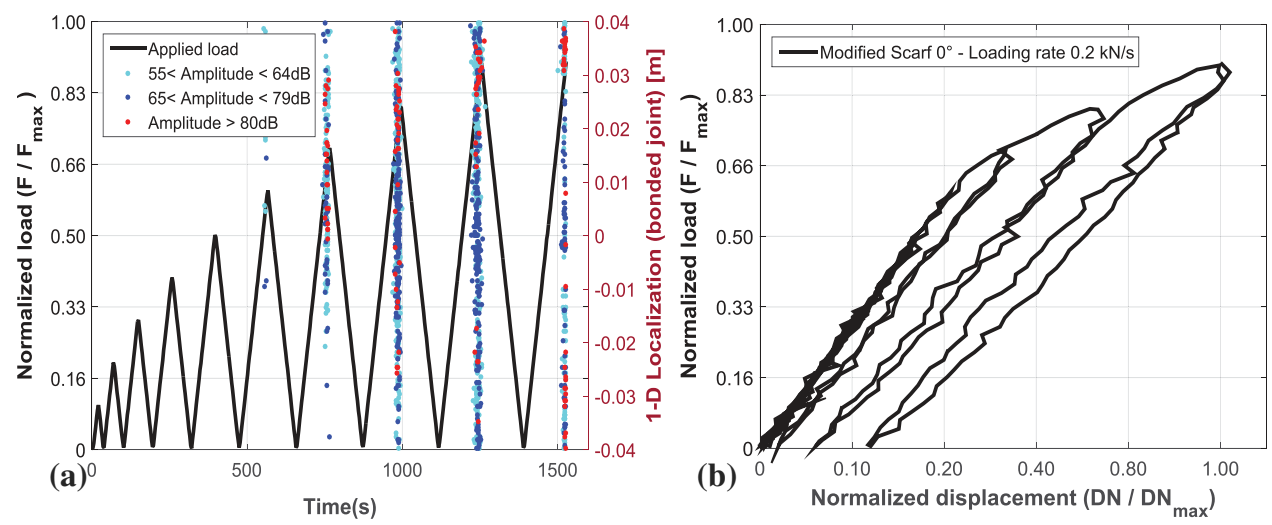

Figure 14. Cycle quasi-static test (a) applied load, (b) mechanical behavior.

\section{Conclusions}

In this study, monotonic tests were conducted in order to firstly investigate the damage evolution of a metal-to-metal adhesively-bonded joint under biaxial loadings (different shear/peel ratios), and secondly to both detect and 
identify acoustic emission (AE) signatures of the different failure mechanisms involved in the bonded joint damage. Specimens with modified scarf joint were manufactured. Three loading configurations were tested (three values of joint angle). In addition to the $\mathrm{AE}$ technique, Digital Image Correlation (DIC) method was used for measuring both the normal and the tangential relative displacements along the adhesively-bonded joint.

Experimental results show that the highest load machine value is obtained with the $45^{\circ}$ modified scarf joint since this loading configuration leads to the highest adhesive bonded surface. Mechanical results also show that the loading configuration strongly influences the normal stiffness value of the bonded joint. These differences in normal stiffness values may be due to the fact that the adhesive bonded surface varies with the loading configuration. The effects of loading rate on the mechanical behavior of adhesive are investigated. Experimental results show that the pseudo-elastic behavior is not affected by the loading rate, whereas the nonlinear regime as well as the load machine at failure is strongly affected by the loading rate.

$\mathrm{AE}$ data was analyzed, and the cluster analysis shows that four cracking mechanisms with different acoustic signatures can be identified from the acoustic activity within the adhesively-bonded joint. However, three main acoustic signatures (cracking mechanisms) were retained. Additional tests by means of tomographic microscopy at different loading stages are in progress in order to identify the defects that generate these particular acoustic signatures.

Finally, an in-depth analysis of AE data was performed in order to try to identify any acoustic signature allowing a detection of the mechanical behavior change during the modified scarf test. The real-time acoustic energy evolution is analyzed, and results show that the highest value of acoustic energy is detected when the slope of the mechanical behavior curve (macroscopic scale) drastically changes. This radical change in slope curve is generally assumed to correspond to the yield strength of the material. Also, the cyclic test showed that the preliminary AE signals (with the highest value of acoustic energy) were linked to an abrupt change of slope which can be related to micro-damage within the adhesive joint. Again, this will be confirmed by RX scans.

\section{Acknowledgements}

The authors thank Li Li (Donghua University, China) and Stephan Lomov (KU Leuven, Belgium) for the cluster analysis tool.

\section{ORCID}

M. Diakhate (D) http://orcid.org/0000-0002-7722-6388 


\section{References}

[1] Vijaya Kumar, R. L.; Bhat, M. R.; Murthy, C. R. L. Some Studies on Evaluation of Degradation in Composite Adhesive Joints Using Ultrasonic Techniques. Ultrasonics. 2013, 53, 1150-1162. DOI: 10.1016/j.ultras.2013.01.014.

[2] Dukes, W. A.; Kinloch, A. J. Non-Destructive Testing of Bonded Joints. Non-Destr. Test. 1974, 7, 324-326. DOI: 10.1016/0029-1021(74)90054-1.

[3] Adams, R. D.; Cawley, P. Defect Types and Non-Destructive Testing Techniques for Composites and Bonded Joints. Constr. Build. Mater. 1989, 3, 170-183. DOI: 10.1016/ 0950-0618(89)90011-1.

[4] Cognard, J. Y.; Davies, P.; Sohier, L.; Créac'hcadec, R. A Study of the Non-Linear Behaviour of Adhesively-Bonded Composite Assemblies. Compos. Struct. 2006, 76, 34-46. DOI: 10.1016/j.compstruct.2006.06.006.

[5] Cognard, J. Y.; Créac'hcadec, R.; Sohier, L.; Davies, P. Analysis of the Nonlinear Behavior of Adhesives in Bonded assemblies-Comparison of TAST and Arcan Tests. Int. J. Adhes. Adhes. 2008, 28, 393-404. DOI: 10.1016/j.ijadhadh.2008.04.006.

[6] Droubi, M. G.; Mcafee, J.; Horne, R. C.; Walker, S.; Klaassen, C.; Crawford, A.; Prathuru, A. K.; Faisal, N. H. Mixed-Mode Fracture Characteristics of Metal-ToMetal Adhesively Bonded Joints: Experimental and Simulation Methods. Procedia Struct. Integr. 2017, 5, 40-47. DOI: 10.1016/j.prostr.2017.07.059.

[7] Nieminen, A. O. K.; Koenig, J. L. Macroscopic and Modern Microscopic NDE Methods for Adhesive-Bonded Structures. Int. J. Adhes. Adhes. 1991, 11, 5-10. DOI: 10.1016/ 0143-7496(91)90054-L.

[8] Hamdi, S. E.; Le Duff, A.; Simon, L.; Plantier, G.; Sourice, A.; Feuilloy, M. Acoustic Emission Pattern Recognition Approach Based on Hilbert-Huang Transform for Structural Health Monitoring in Polymer-Composite Materials. Appl. Acoust. 2013, 74, 746-757. DOI: 10.1016/j.apacoust.2012.11.018.

[9] Schliekelmann, R. J. Non-Destructive Testing of Adhesive Bonded Metal-To-Metal Joints 1. Non-Destr. Test. 1972, 5, 79-86. DOI: 10.1016/0029-1021(72)90099-0.

[10] Korzeniowski, M.; Piwowarczyk, T.; Maev, R. G. Application of Ultrasonic Method for Quality Evaluation of Adhesive Layers. Arch. Civ. Mech. Eng. 2014, 14, 661-670. DOI: 10.1016/j.acme.2013.10.013.

[11] Krause, M.; Dackermann, U.; Li, J. Elastic Wave Modes for the Assessment of Structural Timber: Ultrasonic Echo for Building Elements and Guided Waves for Pole and Pile Structures. J. Civ. Struct. Heal. Monit. 2015, 5, 221-249. DOI: 10.1007/ s13349-014-0087-2.

[12] Diakhaté, M.; Larcher, N.; Takarli, M.; Angellier, N.; Petit, C. Acoustic Techniques for Fatigue Cracking Mechanisms Characterization in Hot Mix Asphalt (HMA). In 7th RILEM International Conference on Cracking in Pavements; Springer Netherlands: Dordrecht, 2012; Vol. 4, pp 771-781. ISBN 9789400745650.

[13] Kowalski, S. J.; Molinski, W.; Musielak, G. The Identification of Fracture in Dried Wood Based on Theoretical Modelling and Acoustic Emission. Wood Sci. Technol. 2004, 38, 35-52. DOI: 10.1007/s00226-003-0211-7.

[14] Huguet, S.; Godin, N.; Gaertner, R.; Salmon, L.; Villard, D., Use of Acoustic Emission to Identify Damage Modes in Glass Fibre Reinforced Polyester. Compos. Sci. Technol. 2002, 62, 1433-1444. DOI: 10.1016/S0266-3538(02)00087-8.

[15] Hwang, H. Y.; Kim, B. J.; Chin, W. S.; Kim, H. S.; Lee, D. G., Prediction of Crack Length and Crack Growth Rate of Adhesive Joints by a Piezoelectric Method. J. Adhes. Sci. Technol. 2005, 19, 1081-1111. DOI: 10.1163/156856105774382453. 
[16] Chen, Z.; Adams, R. D.; Da Silva, L. F. M. Prediction of Crack Initiation and Propagation of Adhesive Lap Joints Using an Energy Failure Criterion. Eng. Fract. Mech. 2011, 78, 990-1007. DOI: 10.1016/j.engfracmech.2010.12.004.

[17] Diakhate, M.; Bastidas-Arteaga, E.; Moutou Pitti, R.; Schoefs, F. Cluster Analysis of Acoustic Emission Activity within Wood Material: Towards a Real-Time Monitoring of Crack Tip Propagation. Eng. Fract. Mech. 2017, 180, 254-267. DOI: 10.1016/j. engfracmech.2017.06.006.

[18] Li, L.; Lomov, S. V.; Yan, X.; Carvelli, V. Cluster Analysis of Acoustic Emission Signals for 2D and 3D Woven Glass/Epoxy Composites. Compos. Struct. 2014, 116, 286-299. DOI: 10.1016/j.compstruct.2014.05.023.

[19] Diakhate, M.; Angellier, N.; Moutou Pitti, R.; Dubois, F. On the Crack Tip Propagation Monitoring within Wood Material: Cluster Analysis of Acoustic Emission Data Compared with Numerical Modelling. Constr. Build. Mater. 2017, 156, 911-920. DOI: 10.1016/j.conbuildmat.2017.09.065.

[20] Droubi, M. G.; Stuart, A.; Mowat, J.; Noble, C.; Prathuru, A. K.; Faisal, N. H. Acoustic Emission Method to Study Fracture (Mode-I, II) and Residual Strength Characteristics in Composite-To-Metal and Metal-To-Metal Adhesively Bonded Joints. J. Adhes. 2018, 94, 347-386. DOI: 10.1080/00218464.2017.1278696.

[21] Dzenis, Y. A.; Saunders, I. On the Possibility of Discrimination of Mixed Mode Fatigue Fracture Mechanisms in Adhesive Composite Joints by Advanced Acoustic Emission Analysis. Int. J. Fract. 2002, 117, 23-28. DOI: 10.1023/A:1022636512843.

[22] de Freitas, S. T.; Zarouchas, D.; Poulis, J. A. The Use of Acoustic Emission and Composite Peel Tests to Detect Weak Adhesion in Composite Structures. J Adhes. 2018. DOI:10.1080/00218464.2017.1396975.

[23] Carrere, N.; Badulescu, C.; Cognard, J.-Y.; Leguillon, D. 3D Models of Specimens with a Scarf Joint to Test the Adhesive and Cohesive Multi-Axial Behavior of Adhesives. Int. J. Adhes. Adhes. 2015, 62, 154-164. DOI: 10.1016/j.ijadhadh.2015.07.005.

[24] Sause, M. G. R. Investigation of Pencil-Lead Breaks as Acoustic Emission Sources. J. Acoust. Emiss. 2011, 29, 184-196.

[25] Nielsen, A. Acoustic Emission Source Based on Pencil Lead Breaking; Svejsecentralen, 1980.

[26] Godin, N.; Huguet, S.; Gaertner, R.; Salmon, L. Clustering of Acoustic Emission Signals Collected during Tensile Tests on Unidirectional Glass/Polyester Composite Using Supervised and Unsupervised Classifiers. NDT E. Int. 2004, 37, 253-264. DOI: 10.1016/J.NDTEINT.2003.09.010.

[27] Li, L.; Lomov, S. V.; Yan, X. Correlation of Acoustic Emission with Optically Observed Damage in a Glass/Epoxy Woven Laminate under Tensile Loading. Compos. Struct. 2015, 123, 45-53. DOI: 10.1016/j.compstruct.2014.12.029. 